\title{
ORQUÍDEAS CENTROAMERICANAS EN INGLATERRA DEL SIGLO XIX
}

\author{
LUIS D. GÓMEZ P. \\ Organización para Estudios Tropicales, Ciudad Científica de UCR y Academia Nacional de Ciencias \\ Apartado 676-2050, San Pedro, Costa Rica. gomezp@ots.ac.cr
}

\begin{abstract}
The study of two major works on orchids of the $19^{\text {th }}$ century in England, the Hortus Veitchii of 1906 and The Orchid Grower's Manual of 1894, show that Central America, here treated to include from Guatemala to western Panama, was an important source of plant materials. Veitch's list is very selective and based, on his opinion, of which plants were outstanding introductions to the trade by his commercial establishment. Williams', on the other hand, is an all-inclusive list, following his wish of "orchids for the million". During the second half of the $19^{\text {th }}$ Cent., English orchidophiles, both trained scientists and amateurs, were growing no fewer than 170 species of Central American provenance. I excluded vague localities such as "Tropical America", and the like even if the species are now known to exist in the area. Of those 170, Costa Rica alone contributed 46 taxa $(27.05 \%)$ to English orchidomania, a mass phenomenon triggered by 1 - the opening of royal institutions such as royal museums and gardens to the general public, and 2- the discovery and popularization of the Wardian case. Soon to be emulated by continental Europe, the Wardian case was short -lived and gave way to more modern, faster, transoceanic travel. Thus commenced the predation for orchid specimens and possibly the decline of plant populations may have contributed to the rarity or disappearance of many species.
\end{abstract}

RESUMEN. Durante la segunda mitad del siglo XIX, Inglaterra experimentó dos eventos significativos en el desarrollo del gusto por las orquídeas y otras plantas: 1- la apertura de instituciones reales o restringidas a estratos superiores de la sociedad inglesa, como museos y jardines botánicos y permitió a las masas participar, vicariamente, de las riquezas del Imperio Británico y 2- el descubrimiento y popularidad de las cajas Wardianas que hicieron posible el transporte de plantas vivas desde confines imperiales y cultivarlas en el viciado aire londinense de la Revolución Industrial. Del estudio de dos obras importantes, el Hortus Veitchii de 1904 y el Orchid Grower's Manual de 1894, la primera orientada a una clientela pudiente y la otra a lo que su autor anhelaba, "orquídeas para todos", se desprende que no menos de 170 especies de orquídeas centroamericanas eran cultivadas en Inglaterra. De esas, 46 (27.05\%) provenían de Costa Rica. La orquideomanía inglesa, pronto emulada por la Europa continental, y la caja de Ward, pronto sustituida por transportes transoceánicos más rápidos, también precipitaron el despoje de plantas y puede haber contribuido a la rareza o extinción de algunas especies.

Palabras clave/ Key Words: Orchids, Central America, Horticulture, England, Nineteenth Century.

Hace algunos meses y motivado por simple curiosidad, inicié una lectura cuidadosa del Hortus Veitchii (Veitch 1906) para establecer cuántas plantas costarricenses habían sido introducidas a la horticultura europea por ese establecimiento comercial. Esas introducciones fueron varias pero, relativamente, fueron las orquídeas el grupo más importante. Decidí así dedicar el primer análisis a esa familia de plantas ampliando la cobertura al área centroamericana que para estas notas se extiende desde Guatemala hasta Panamá inclusive, en virtud de que en la época que interesa, gran parte de la Provincia de Chiriquí de ese país, fue parte del territorio costarricense hasta 1903, año en que se separó Panamá de la Gran Colombia y se intentó fijar los límites actuales entre los dos países, empresa no libre de conflictos. Además, la región de Chiriquí y las tierras limítrofes de la Costa Rica de hoy, conforman una unidad biogeográfica indiscutible y esas zonas fueron importante escenario de acción de los coleccionistas de plantas y botánicos de la época.

Iniciado el recuento, fue evidente que debía recurrir a otras fuentes y escogí para ello el summum de ese período de la historia, la obra The Orchid-Grower's Manual de Williams \& Williams (1894). Como obras 
de consulta, Allen (1949), Ames (1937), Ames \& Correll (1952, 1953), Dressler (1980,1993, 2003), Hamer (1974a, 1974b, 1981, 1982, 1983, 1984a, 1984b, 1985, 1988, 1990, 2001), Hawkes (1965), Pupulin (2002), Williams $(1946,1951,1956)$ y otras citadas en el texto.

Aunque las orquídeas en Europa se han conocido desde la antigüedad, Teofrasto ( \pm 370-285 AC), menciona especies de Orchis y les atribuye algunas propiedades medicinales, su cultivo como ornamentales no parece haber tenido mayor importancia y hasta finales del siglo XVIII no pasaron de ser interesantes para algunos científicos y naturalistas. Situación impensable para un siglo importantísimo para la botánica porque se puede decir que de 1809 a 1851 se llegó a una teoría unitaria sobre la estructura y los ciclos de vida de las plantas y de 1851 a 1912 se establecieron las bases de la Botánica como ciencia moderna (Bernal 1983, Coleman 1977, Morton 1981, Nordenskiöld 1928, Singer 1950) en las elitistas y ebúrneas torres de los científicos. Pero, ¿Y el resto de la población? Dos eventos habrían de cambiar esa situación.

El primero de ellos fue la apertura a las masas de museos y jardines botánicos, hasta entonces dominio exclusivo de la aristocracia y estratos superiores de la sociedad inglesa, lo que permitió al gran público incorporarse, vicariamente, al apogeo del Imperio Británico en tiempos de la reina Victoria, observando, conociendo y aprendiendo sobre los "productos exóticos" de los confines imperiales. Debo recalcar aquí que el interés de los administradores del Imperio no eran científicos sino utilitarios, v.g. algodón, amapolas (opio), té, hule, azúcar, medicinales (quinina, que abriría el Raj de la India y el África a la colonización inglesa), etc. (Musgrave \& Musgrave 2000) hasta incluso bloquear cualquier conocimiento sobre anticonceptivos que mermaran una creciente y adquisitiva población (Schiebinger 2004). El primer museo en abrir fue el Hancock, de Newcastle, que a partir de 1835 organizó horas de visita vespertinas. Pero el Hancock en Newcastle no era el corazón del Imperio, Londres y su British Museum con su renacentista concepto de artes y ciencias, lo eran. Allí, el ambiente para admitir a la clase trabajadora era negativo. Teóricamente abierto "para toda persona de buena apariencia”, solo abría tres días por semana de $10 \mathrm{am}$

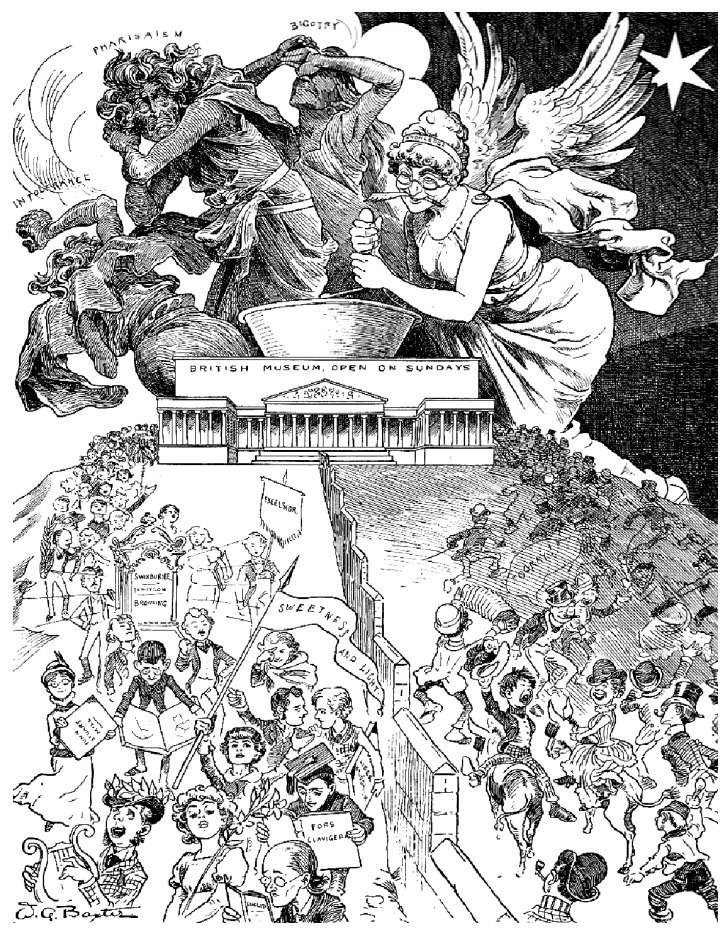

Figura 1. "A Dream of the Future-Sunday Opening" del Anuario Judy, 1885. A la derecha la "canalla inglesa" que sale ilustrada después de la visita. Una Musa alada expulsa a la hipocresía, el fariseismo y a la intemperancia, (col. del autor).

a 4 pm, horario que excluía a la abrumadora mayoría de la población londinense, fuerza motora de la Revolución Industrial, y que de variadas formas exigía acceder a la cultura (fig. 1). Cuando, en 1835 una comisón presentó la posibilidad de abrir a un mayor y diverso público en ciertas fechas del año, e.g. la semana de Pascua, Sir Henry Ellis, Bibliotecario Principal del Museo Británico, dijo entre otras cosas (el subrayado es mío): "The most mischievous portion of the population is abroad and about at such a time...the more vulgar class would crowd into the Museum... sailors from the dock yards and girls whom they might bring with them...The more important part of the population...would be discontented" (Fig. 2). Richard Owen, opositor encarnizado de Darwin, hizo eco a esas palabras y esnobismo cuando en 1840 visitó los Museos de Nottinghan y Leicester en un ensayo de apertura a las masas escribió a su esposa (el subrayado es mío): "Never saw a better experiment of the amount of danger to be apprehended for indiscriminate admission of English 


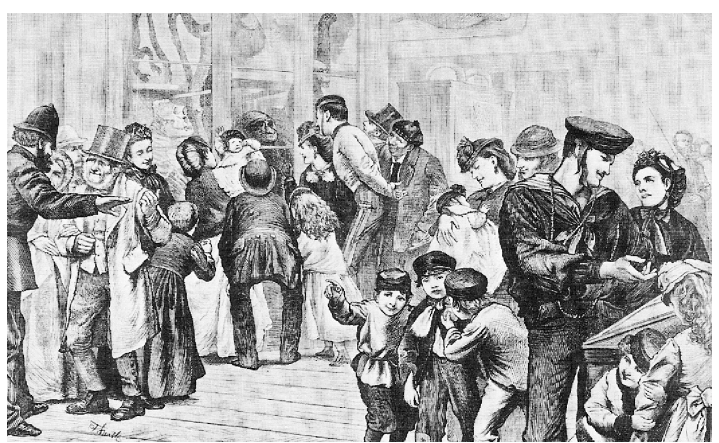

FigurA 2- "Holiday Time at the British Museum" por F. Smith (1873). A la derecha, un marinero con su "chica", visión temida por Owen (col. del autor).

canaille..." (Barber 1980). No fue sino hasta 1879 que el Museo Británico abrió regularmente todos los días en horario exclusivo y hasta 1896 , cuando el Departamento de Historia Natural se trasladó a South Kensington (separación de las Ciencias Naturales del arte, antropología, etc.), que permitió el ingreso "a personas de buena apariencia", los domingos. Ese cambio de actitud, eliminando o atenuando el elitismo a favor de la educación de la multitud, constituyó el fundamento del naturalismo inglés que eventualmente rindió abundantes frutos a las Ciencias Biológicas. Iguales ritos de pasaje sufrió el Real Jardín Botánico de Kew, más o menos simultáneamente.

Establecido en 1759 en terrenos de S.A.R. Augusta, Viuda Princesa de Gales, madre de Jorge III, Kew se mantuvo cerrado al público por 82 años hasta 1841 en que pasó a manos de gobierno inglés con William $J$. Hooker como su primer Director. En la lista de plantas cultivadas allí, compilada por Aiton en 1789 y conocida como Hortus Kewensis (Aiton 1789), se enumeran quince especies de orquídeas, entre ellas dos neotropicales posiblemente provenientes de Jamaica, Epidendrum fragrans y Epidendrum cochleatum, que pasaron a ser Encyclia, y hoy especies de Prosthechea. Para 1813 el número de orquídeas había aumentado a cuarenta y seis. Como indiqué al inicio de esta introducción, hasta 1800 el cultivo de orquideáceas en Europa no era de importancia. En Inglaterra, la Sociedad Londinense de Horticultura se interesó en las orquídeas a partir de 1809 y, en relativo corto tiempo ese grupo de plantas habría de alcanzar una enorme relevancia, a diferencia de otras naciones pujantes como los Estados Unidos de
América donde las primeras orquídeas fueron cultivadas hasta $c a .1830$ por John Wright Boott, en Boston. En Inglaterra, fue la floración de Cattleya labiata Lindley, recogida en 1818 en la Sierra de los Órganos, Brasil, en el orquidario de William Cattley a quien John Lindley dedicó el género, aunque un congénere, entonces llamado Epidendrum violaceum, procedente de S. Paulo, Brasil, recibido en 1810 por el Jardìn Botànico de Liverpool, floreció el año siguiente pero no fue descrita como Cattleya loddigesii sino hasta 1819 cuando Lindley estableció el género. La C. labiata por décadas considerada la más exquisita de las orquídeas, marcó el inicio de la orquideología y, en buena medida, fue el arrobo del Sexto Duque de Devonshire, William Spencer Cavendish, con una planta de Oncidium papilio (Psychopsis papilio), hizo del pasatiempo de los "orchidists" una actividad de moda, pero limitada a la aristocracia y las clases pudientes ya que los precios de las plantas procedentes de las regiones remotas alcanzaban precios altísimos (Reinikka 1995). Las orquídeas pronto fueron sinónimos de exoticidad en el Nueva Mundo, incluso apareciendo en pinturas neoclásicas del período (Fig. 3 ). Pronto se establecieron casas comerciales dedicadas a la búsqueda, cultivo y venta de orquídeas y otras plantas exóticas que enviaron un ejército de recolectores de todos los rincones del globo. Pero estos amagos de comercialización se toparon con un enorme y oneroso problema: muy pocas plantas llegaban a Inglaterra en buen estado, la mayoría no soportaban el largo viaje marino.

Es aquí donde entra en escena el segundo evento más importante en el desarrollo de la orquideología como pasatiempo de muchos y fuente de nuevas especies para los taxónomos. Me refiero al descubrimiento en 1829 , por lo demás fortuito, del Dr. Nathaniel Bagshaw Ward, connotado naturalista de quien se dice era poseedor de una colección de unas 25.000 plantas y que, además, era aficionado a los lepidópteros, que criaba desde sus fases más tempranas. En cierta ocasión, colocó una crisálida dentro de una botella para protegerla del viciado aire londinense. Cuando transcurrió el tiempo de extraer el insecto emergido, con sorpresa descubrió que habían germinado dos plantas, una gramínea y un helecho, que mantuvo en la botella por muchos años e hicieron su début público en la Gran Exhibición de 1851. Ward 


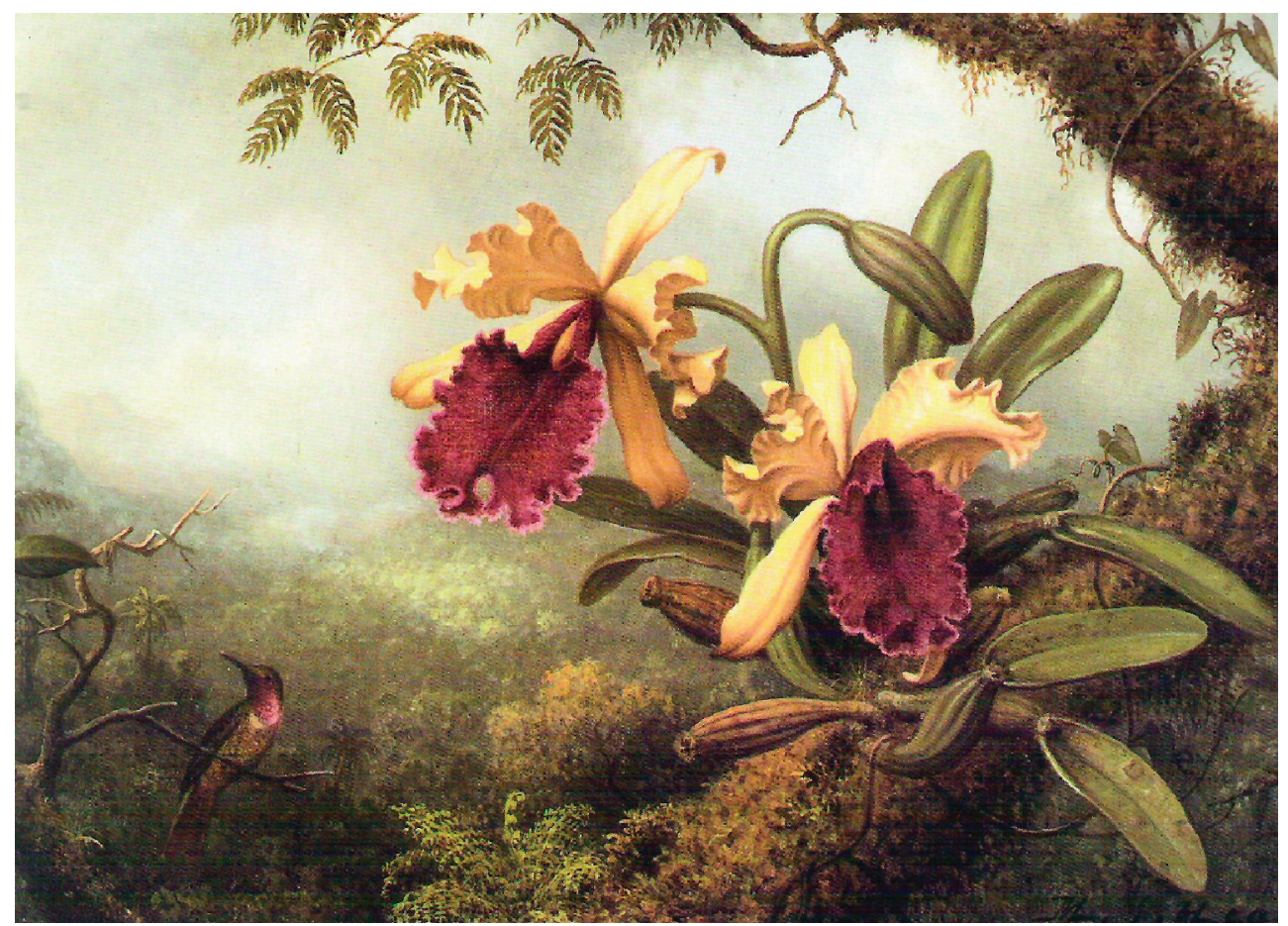

Figura 3. Orquídeas y colibríes (detalle), 1875-1885. De Martin Johnson Heade. Óleo sobre tela (141/2 x 221/4 ").

Museo de Bellas Artes, Boston, Mass., E.E.U.U.

experimentó con muchas botellas y muchas plantas y llegó a cultivar exitosamente unas decenas de ellas que no habrían crecido expuestas al ambiente exterior. Comunicó su pequeño experimento a George Loddiges, propietario de la famosa Loddiges Nursery en Hackney, quien de inmediato se percató del potencial comercial del descubrimiento de Ward y construyó recipientes similares para enviar de Londres a Australia un pedido de plantas. En noviembre de 1834 su corresponsal en las antípodas le comunicó que el envío había llegado a destino con las plantas vivas y prósperas. Así hizo su espectacular entrada en escena la caja wardiana. Ward publicó un libro en 1842, On the growth of Plants in Closely Glazed Cases, pero nunca he tenido oportunidad de leerlo. El hijo de William Hooker, de Kew, Joseph Dalton Hooker, usó cajas wardianas para enviar a Inglaterra plantas de Nueva Zelandia, durante el pionero viaje del HMS Erebus en la circumnavegación de Antártica. Rápidamente, las cajas wardianas se convirtieron de simples y prácticas cajas de vidrio en mobiliario común en las casas de los aficionados y científicos (Fig. 4). ¡Me llama la atención que exis- tiendo ya los acuarios, nadie pensara en terrarios antes de la observación del Dr. Ward!

Pero con la caja wardiana también llegó un aspecto negativo y es que con el problema del tránsito marino resuelto, los recolectores a sueldo sobreexplotaron el recurso orquídea casi hasta la extinción de las especies más cotizadas. Por ejemplo, en la frenética búsqueda de Miltonia vexillaria [Miltoniopsis vexillaria (Reichb.f.) God.-Leb.], los hábitats naturales de la especie parecían el resultado de incendios forestales. ¡En Colombia, por ejemplo, la "cacería” de 10.000 especímenes de Odontoglossum crispum [O. crispum Lindl.] resultó en la tala de 4.000 árboles! Transcribo un párrafo de una carta de Carl Johannsen desde Medellín a su patrocinador: "I shall dispatch tomorrow 30 boxes, 12 of which contain the finest of all the Aureas, (Cattleya aurea Lind1.) the Monte Coromes form, and 18 cases containing the great Sanderiana [C. sanderiana hort, C. gigas Lind. \& André] type all collected ... They are now extinguished in this spot...I have finished all along the Rio Dagua where there are no plants left..." (fide Reinikka, 1995). Con la caja wardiana, florecieron los 


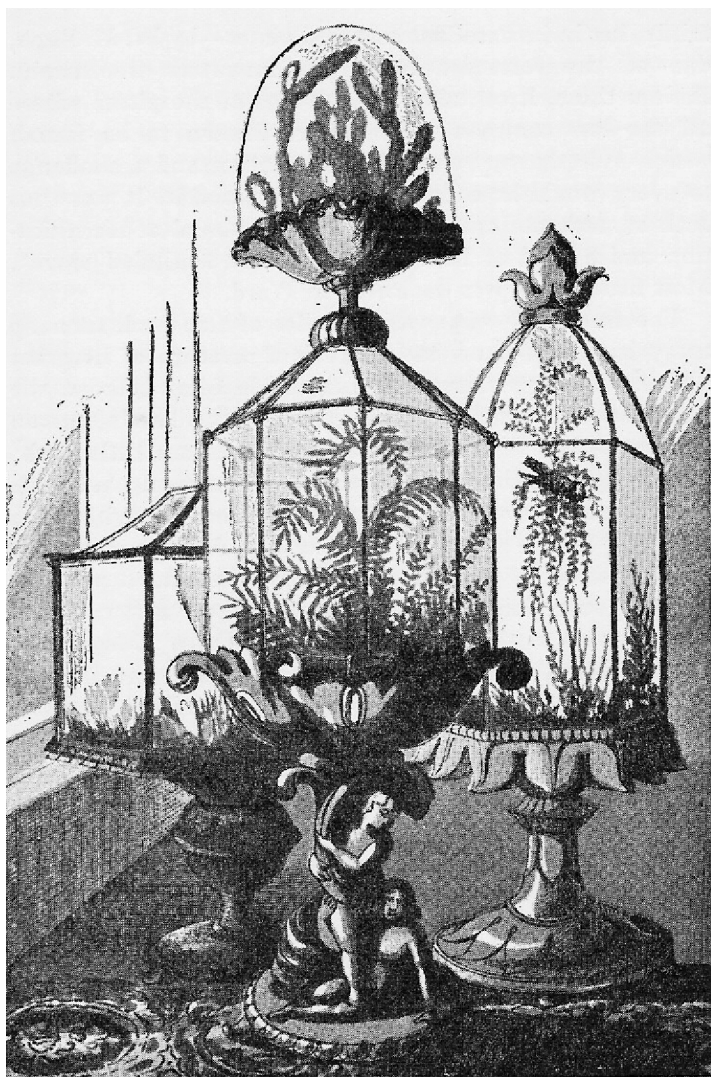

FigurA.4- Cajas wardianas para el hogar. Tomado de The New Practical Window Gardener, catálogo para 1877, de J.R. Mollison (col. del autor).

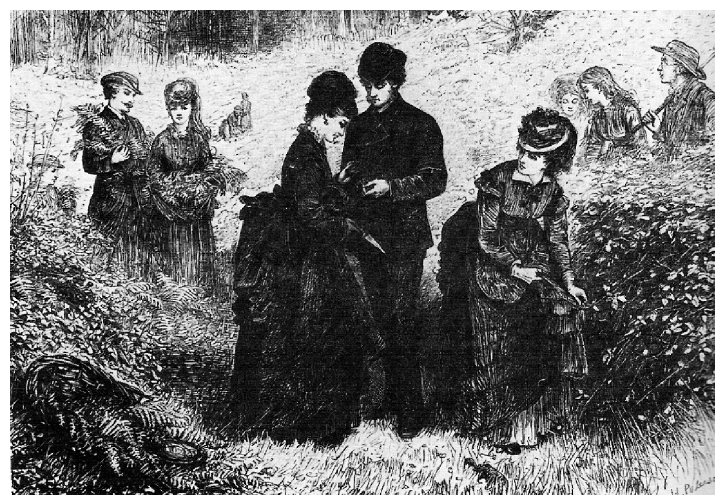

FIGURA 5 - "Gathering Ferns", de Illustrated London News, 1877. La pteridomanía en su zenit (col. del autor).

viveros comerciales y también se suscitaron dos histerias colectivas: la orquideomanía y la pteridomanía. Sobre la pteridomanía (Fig.5) una simpática obra, The Victorian Fern Craze, de Allen (1969).
De los establecimientos comerciales de mayor impacto escogí la casa de James Veitch en Chelsea y la de B.S. \& H. Williams, Victoria and Paradise Nurseries, Upper Holloway, ambas en Londres, y que produjeron la primera un catálogo Hortus Veitchii y la segunda un vademécum para los orquideómanos, el famoso The Orchid-Grower's Manual.

El Hortus (Fig. 6) es una edición para circulación privada y de pocos ejemplares elegantemente empastada y que utilizó un papel para acuarela de gran peso. La edición está obviamente hecha para una selecta y adinerada lista de clientes. La obra, con un total de 541 páginas con 50 ilustraciones de fotograbado (Fig. 7), menciona un total de 1971 taxa de los cuales 1572 corresponden a otros grupos de plantas, y esta dividida en cinco secciones o capítulos. La primera es una historia de la familia Veitch y la creación de la empresa y su evolución. Fundada por John Veitch (1752-1839) fue adquirida por James Veitch y su hijo homónimo en 1853 lo que dio origen a John Veitch \& Son. John Veitch hijo estuvo a cargo de la operación en Chelsea.

La segunda parte está destinada a cortas biografías, con aportes interesantes, de los recolectores viajeros patrocinados por Veitch \& Son que suman 22 entre 1840 y 1905 . De ellos cuatro recorrieron el área centroamericana: Carl Kramer en 1867 que estuvo en Guatemala y Costa Rica pero resultó “..unsuitable for the work" (sic, pág.55) y a el se debe el descubrimiento de Ticoglossum krameri (Rchb.f.) Halbinger (Odontoglossum krameri hort.) y de Psychodes kramerianum (Rchb.f.) Halbinger(Oncidium kramerianum hort.).

Gottlieb Zahn entre 1869 y 1870 recorrió parte de Centroamérica en busca de helechos y orquídeas. Su meta era el Oncidium warsczewiczii Rchb.f. pero no tuvo éxito, el triunfo correspondió a Endrés y la especie le fue dedicada como Miltonia endresii Nichols. Luego de recolectar en Chiriquí trató de viajar a San José pero murió ahogado en el intento. Se le conmemora en una bromelia, Guzmania zahnii (Hook.f.) Mez.

George Downton, al servicio de Veitch entre 1870 y 1873, recorrió América del Sur, y las Islas de Juan Fernández. En 1870 llegó a Costa Rica para colaborar con Endrés pero sólo permaneció unos meses.

A. R. Endrés, recomendado a Veitch por Bateman, 


\section{HORTUS VEITCHII}

\section{A HISTORY}

OF THE

RISE AND PROGRESS OF THE NURSERIES OF MESSRS, JAMES VEITCH AND SONS, TOGETHER WITH AN ACCOUN' OF THE BOTANICAL COLLECTORS AND HYBRIDISTS EMPLOYED BY THEM AND A LIST OF THE MOST REMARKABLE OF THEIR INTRODUCTIONS

JAMES H. VEITCH

3llugtrated with fifte Dhotogravure Dlates

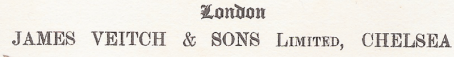

FIgURA 6. Portada del Hortus Veitchii, edición príncipe, 1906 (col. del autor).

colaboró con George Ure-Skinner en Guatemala y al deceso de Zahn le ordenaron trasladarse a Costa Rica en 1871 en busca del Odontoglossum warsczewiczii (v. supra), Cattleya dowiana y Anthurium scherzerianum, en gran demanda y permaneció en Costa Rica hasta entrado 1873. Se le han dedicado varias especies de orquídeas y fue un extraordinario recolector de helechos. George Downton fue el encargado de acompañar sus colecciones a Inglaterra. Atwood (1999, fide Ossenbach 2003) dice que murió asesinado en Riohacha, Colombia.

La tercera sección está dedicada a datos biográficos de los principales horticultores, la mayoría de ellos dedicados a la hibridización de orquídeas. No se discuten en estas notas.

La cuarta sección nos interesa. Se intitula Orchid Species. A list of the principal orchid species introduced by Messrs. Veitch. En la lista se enumeran 219 especies con su autor, procedencia, a veces datos curiosos y una muy somera descripción. De esas

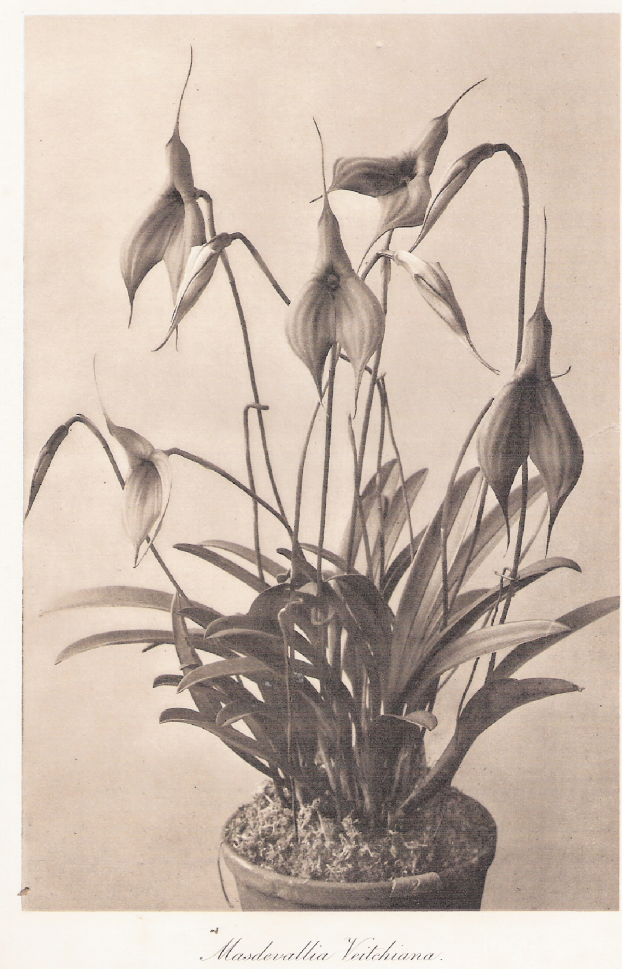

Figura 7. - Masdevallia veitchiana, descubierta en los Andes peruanos por Pearce en 1866 y descrita por Reichenbach en 1868. Fotograbado (frente pág. 140) del Hortus Veitchii.

especies $23(10.5 \%)$ son centroamericanas y de ellas 17 ( $73.9 \%$ ) procedían de Costa Rica. Cito verbatim el texto correspondiente (Pág. 116-117) a una de nuestras especies como ejemplo del contenido: "Cattleya dowiana, Batem.-Syns. C. lawreanceana, Warsc. ; C. labiat, var. Dowiana, Veitch - Gard. Chron. 1866, p. 922; Bot. Mag. T. 5618; Fl. Des Serres, tt. 1709-1710; L'Illus. Hort. T. 525; The Garden, 1877, vol. XII. T. 99; Veitch's Man. Orch. Pl. pt. Ii. P. 16 - This superb orchid flowered for the first time in this country at Chelsea in the autumn of 1865. The plants were obtained through Mr. G. UreSkinneri, whose collector Mr. Arce, a zealous naturalist, had obtained them in Costa Rica. Plants had previously been sent to this country in 1850, but arriving in a bad condition, had all died without flowering. It was the wish of Warszcewicz, the original discoverer, that his plant should bear the name Lawrenceana, in compliment to Mrs. Lawrence of Ealing, a generous patroness of Horticulture, but as his specimens mis- 
Cuadro 1. Orquídeas Centroamericanas en Hortus Veitchii.

\begin{tabular}{|c|c|c|}
\hline Nombre del Hortus Veitchii & Pág. & Nombre actual \\
\hline Cattleya bowringiana Veitch & 116 & Guarianthe bowringiana (Veitch) Dressler \& Higgins \\
\hline C. dowiana Bat. * & 116 & C. dowiana Bat. \\
\hline C. skinneri Bat. v. alba Rchb.f. * & 117 & Guarianthe skinneri (Bat.) Dressler \& Higgins \\
\hline E. lindleyanum (Bat.)Rchb.f. * & 134 & Barkeria lindleyana Bat. ex Lindl. \\
\hline E. pseudepidendrum Rchb.f. * & 134 & E. pseudepidendrum Rchb.f. \\
\hline Laelia anceps Lindl. & 136 & Laelia anceps Lindl. \\
\hline Lycaste skinneri Lindl. & 137 & Lycaste skinneri Lindl. \\
\hline Masdevallia attenuata Rchb.f. * & 137 & Masdevallia attenuata Rchb.f. \\
\hline M. lata Rchb.f. & 138 & M. lata Rchb.f. \\
\hline M. reichenbachiana Endrés * & 139 & M. reichenbachiana Endrés \\
\hline M. triaristella Rchb.f. * & 139 & Trisetella triglochin (Rchb.f.) Luer \\
\hline Maxillaria. ctenostachya Rchb.f. * & 140 & Maxillaria ctenostachya Rchb.f \\
\hline Miltonia endresii Nicholson * & 140 & Miltoniopsis warsczewiczii ( Nicholson)Garay \& Dunst. \\
\hline Mormodes fractiflexum Rchb.f. * & 141 & Mormodes fractiflexa Rchb.f. \\
\hline M. skinneri Rchb.f. & 141 & M. skinneri Rchb.f. \\
\hline Notylia albida Klotzch & 142 & Notylia barkeri Lindl. \\
\hline Odontoglossum krameri Rchb.f. * & 143 & Ticoglossum kramerii (Rchb.f.)Halbinger \\
\hline O. oerstedii Rchb.f. * & 144 & T. oerstedii (Rchb. f.) Halbinger \\
\hline O. uro-skinnerii Lindl. & 144 & Rhynchostele uro-skinneri (Lind1.) Lindl. \\
\hline Oncidium bryolophotum Rchb.f. & 145 & Oncidium bryolophotum Rchb.f. \\
\hline O. warscewiczii Rchb.f. * & 146 & O. warscewiczii Rchb.f. \\
\hline Polycynis gratiosa Endrés \& Rchb.f. (sic) * & 150 & Polycycnis gratiosa Endrés \& Rchb.f \\
\hline Rodriguezia leochilina Rchb.f. * & 151 & Hybochilus lochilinus (Rchb.f.) M.W. Chase \\
\hline Trichopilia marginata Henfr. $v$. lepida Veitch * & 155 & Trichopilia marginata Henfr. $v$. lepida Veitch \\
\hline Zygopetalum burtii Benth. * & 158 & Huntleya burtii Rchb.f. \\
\hline
\end{tabular}

* Especies recogidas en Costa Rica

carried, this fact was not made known until after Bateman had named it in compliment to Captain J. M. Dow of the American Packet Service, to whose kindness orchidists and men of science owe so much. It has proved potent as a parent by hybridizing, many fine seedlings now in cultivation being due its influen$c e$ ". Las especies e híbridos de orquídeas suman 521 ( $6.4 \%$ del total, incluídos los sinónimos). En el Cuadro 1, la lista de especies centroamericanas del Hortus Veitchii y su sinonímia.

La quinta sección trata de 302 híbridos de orquídeas introducidos por Veitch a la horticultura británica pero no me adentro en ningún análisis toda vez que no se indica la procedencia de los progenitores, algunos obviamente centroamericanos, y es un laberinto de nombres, muchos de fantasía, otros hortíco- las y sin validación botánica, de un grupo de plantas que a su voluptuosa y concupiscente apariencia suman una venalidad y libertinaje genético, proverbiales. La sexta parte se dedica a otros grupos de plantas y trataré las entidades costarricenses en otra oportunidad y lugar.

Debido a que la lista de orquídeas del Hortus Veitchii es parcial, como indica el subtítulo de la sección, lista de las orquídeas principales (según Veitch), recurrí a la obra Orchid-Grower's Manual, de Benjamin Samuel Williams y su hijo Henry William Williams, publicada en 1894 en su séptima y última edición (Fig. 8). El Manual para Orquideólogos, basado en las plantas cultivadas en el establecimiento comercial de los Williams, Victoria \& Paradise Nurseries, tiene 796 páginas, 310 ilustra- 


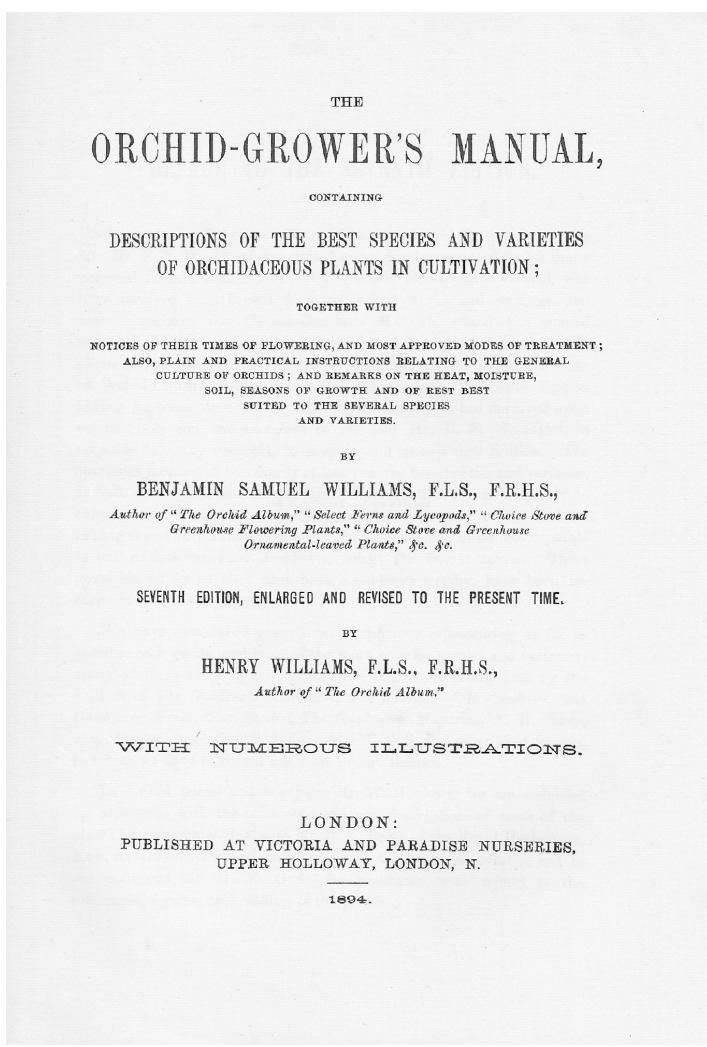

Figura 8. - Portada de Orchid Grower's Manual de Williams \& Williams, 7a. edición de 1894 (col. del autor).

ciones de línea. Las primeras 73 páginas dedicadas a generalidades del cultivo y a partir de una breve discusión sobre el precio de las plantas inicia una enumeración alfabética de géneros y especies con un total de 3359 taxa de los cuales los autores del Manual consideran 2650 como válidas y el resto, 709 , como sinónimos. De las especies válidas, 163 $(6.15 \%)$ corresponden a especies centroamericanas y de ellas 39 (23.9\%) son costarricenses porque así lo indican. Por imprecisas, omito aquellas para cuya procedencia se dice "Tropical America", "Central America", a sabiendas de que se encuentran en nuestro país. Del gran total de 3359 taxa, $702(20.8 \%)$ son especies de A?rides (606 spp.), Vanda (677 spp.), Disa (702 spp.), Dendrobium y Cypripedium s. latissimus (para incluir Calypso, Paphiopedilum, Phragmidium, Selenipedium, etc.) (542 spp.) que representan el $20.8 \%$ de los taxa, muestra indiscutible de la ominipresencia del Imperio desde sus cua- tro puntos cardinales. En el Cuadro 2, la lista de especies centroamericanas y su sinonímia. Los Williams fueron grandes promotores de la orquideomanía inglesa, en particular Benjamin quien inició en 1851 a sugerencia de John Lindley una serie divulgativa titulada Orchids for the Millions en el Garden Chronicle. Un derivado de esa serie fue su famoso Orchid Album (Warner \& Moore 1882-1897) una publicación seriada mensual de cromolitografías basadas en dibujos de John Fitch, y de la cual reproduzco cuatro láminas (Fig. 9).

Debido a la naturaleza de la selección, en Veitch con miras a una clientela selecta y de plantas en su opinión principales, y de una actitud populista, de orquídeas para los millones, de los Williams, sería inútil cualquier análisis o comparación numérica. Sí es digno de comparar los criterios taxonómicos de ambos establecimientos. Veitch, por ejemplo, cita a Cattleya skinneri y una forma alba. Los Williams asignan a esa especie de Guarianthe varios nombres varietales. En algunos taxa, como Lycaste skinneri, hasta 15 formas varietales!. Ambas obras tienen un sinnúmero de especies y variedades del género Cattleya, que en opinión de los estudiosos del género, son solamente unos pocos taxa válidos, desde unas decenas (Hawkes 1965), hasta poco más de veinte (Withner 1988). Williams \& Williams (pág.407) hacen un interesante comentario sobre las especies de Gongora, en particular G. maculata, y lo que llaman "complejo de G. quinquinervis de Reichenbach”, grupo aún confuso. Aún haciendo caso omiso de los incipientes conceptos genéricos de la época, ambas son importantes para vislumbrar el impacto de la apertura popular de las instituciones reales y la importancia de la caja wardiana en el desarrollo de la orquideomanía en la Inglaterra decimonónica y de la riqueza, tanto en diversidad como en atractivo, de la flora centroamericana. Las plantas costarricenses jugaron un papel muy importante en el desarrollo temprano de la orquideología amateur y profesional. Eliminando las duplicaciones en ambas listas, 46 especies costarricenses eran cultivadas en Inglaterra hacia el cambio de siglos. La orquideomanía inglesa, emulada en corto tiempo por europeos continentales y la caja wardiana, contribuyeron, posiblemente, a la extinción o actual rareza de muchas especies. 

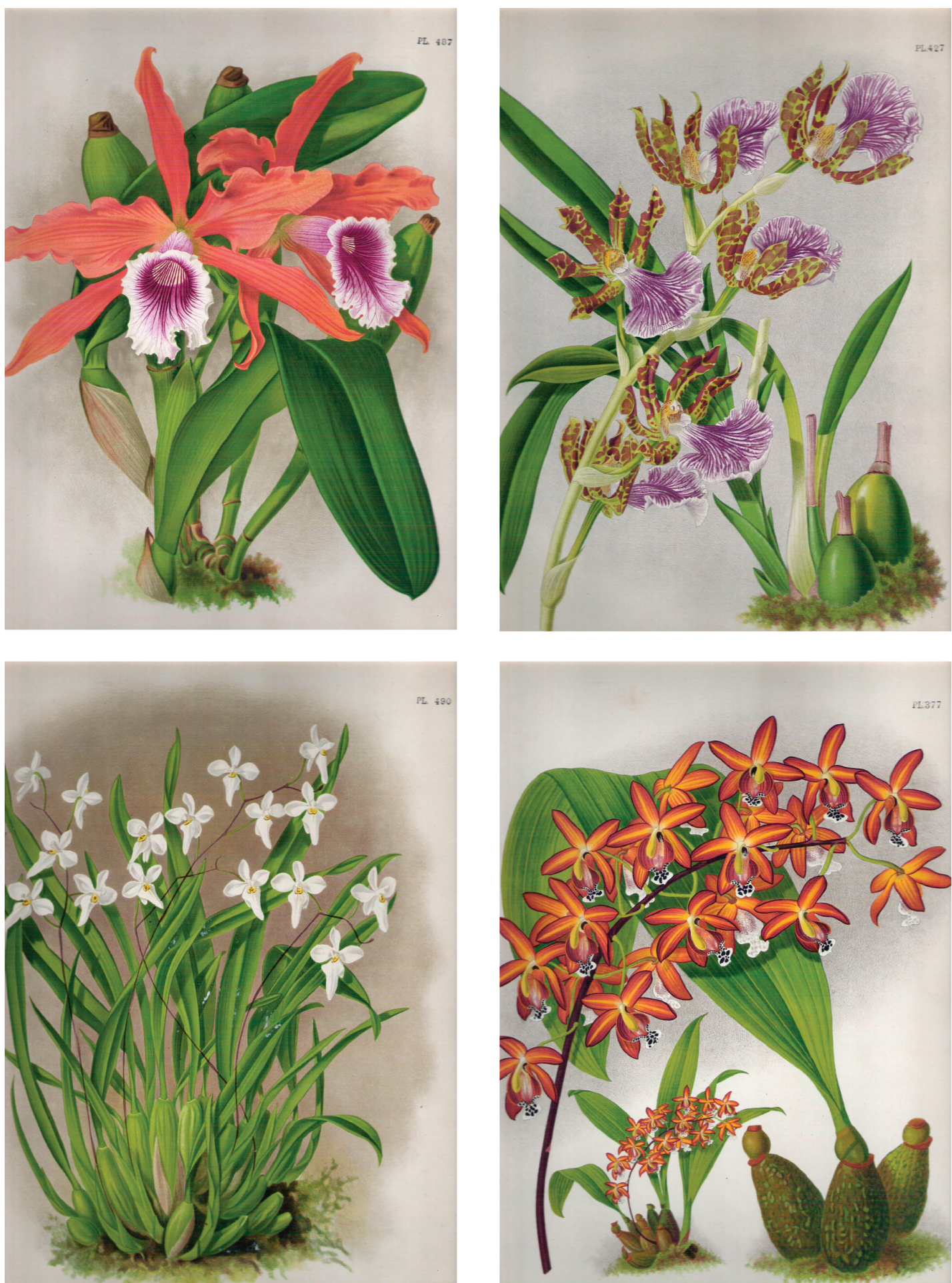

FIGURA 9 - Arriba, izquierda, Laelia grandis tenebrosa (Rolfe) [Sophronitis tenebrosa (Rolfe) Berg. \& Chase]; arriba, derecha, Zygopetalum mackayi Hooker; abajo, izquierda, Palumbina candida Rchb.f.; abajo, derercha, Eriopsis rutidobulbon Hooker. Láminas 467, 427, 490, 377, del Orchid Album de B.S. Williams, sin fecha (Col. del autor). 
Cuadro 2. Orquídeas Centroamericanas en The Orchid-Grower's Manual.

\section{Nombre en Grower's Manual}

Acineta densa Lindl.

Acropera armeniaca Lindl.

Anoectochilus eldorado hort.

Barkeria lindleyana Batem.*

Barkeria lindleyana Bat. v. centerae Endrés \& Rchb.f. *

Barkeria melanocaulon Rich. \& Gal. *

Barkeria skinneri Batem. *

Barkeria skinneri v. superba hort

Barkeria spectabilis Batem.

Batemania burtii Endrés \& Rchb.f.*

Batemania wallisii Rchb.f. *

Batemania wallisii v. major Rchb.f*

Brassavola acaulis Lindl.

B. dygbiana Lindl.

B. glauca Lindl.

B. lineata $\mathrm{Hk}$.

B. venosa Lindl.

Brassia gireoudiana Rchb.f. \& Warsc. *

B. guttata Lindl.

B. lawrenceana Lindl. v. longissima Rchb.f.

B. verrucosa Lindl.

B. verrucosa Lindl. v. grandiflora Wms.

Catasetum scurra Rchb.f.

Cattleya aurea sine auct in opus (Linden?)

\section{C. bowringiana Veitch}

C. dowiana Batem. *

C. granulosa Lindl.

C. guatemalensis Moore

C. guatemalensis v. wischusseniana Rchb.f. *

C. skinneri Batem. *

C. skinneri Batem. v. alba Rchb.f. *

Chysis limminghei Rchb.f.

Coelia bella Rchb.f. (sic)

Cycnoches aureum Lindl.

C. barbatum Lindl. *

C. ventricosum Batem.

Cypripedium caudatum Lindl.

C. caudatum f. Louxenbourg hort.

C. caudatum Lindl. v. roseum hort.

C. warscewiczii hort.

C. longifolium Warscz. \& Rchb.f

C. irapeanum LLlav. \& Lex.

Epidendrum aromaticum Batem.

\section{Pág. Nombre actual}

75 Acineta densa Lindl. \& Paxt.

77 Gongora armeniaca (Lindl. \& Paxt.) Rchb.f

109 Goodyera sp.

118 Barkeria lindleyana Batem.

118 Barkeria lindleyana Batem.

118 Barkeria lindleyana Batem. ?

118 Barkeria lindleyana Batem. ?

118 Barkeria lindleyana Batem. ?

119 Barkeria lindleyana Batem. ?

120 Huntleya burtii (Endrés \& Rchb.f.) Pfitzer

121 Huntleya burtii (Endrés \& Rchb.f) Pfitzer Huntleya burtii (Endrés \& Rchb.f) Pfitzer

126 Brassavola acaulis Lindl. ex Pax.

127 Rhyncholaelia digbyana (Lindl.) Schlechter

127 R. glauca (Lindl.) Schlechter

127 Brassavola acaulis Lindl. ex Paxt.

128 Brassavola nodosa (L.) Lindl.

129 Brassia gireoudiana Rchb.f. \& Warsc.

129 B. maculata R. Br. in Aiton

130 B. caudata (L.) Lindl.

131 B. verrucosa Lindl.

131 B. verrucosa Lindl.

149 Clowesia warscewitzii (Lindl.ex Pax.) Dodson

153 Cattleya dowiana Bat. v. aurea Wms. \& Moore, non Linden fide Williams

154 C. bowringiana Veitch

160 C. dowiana Batem.

165 C. granulosa Lindl.

165 C. patinii Cogn.

165 Guarianthe patinii (Cogn.) Dressler \& Higgins

185 Guarianthe skinneri (Bat.) Dressler \& Higgins

Guarianthe skinneri v. alba Rchb. $f$.

196 Chysis bractescens Lindley

201 Coelia bella (Lem.) Rchb.f.

215 Cycnoches egertonianum Batem.

215 Mormodes hookeri Lem.

217 C. ventricosum Baemt.

240 Cypripedium caudatum Lindl.

241 Cypripedium caudatum Lindl.

241 C. caudatum Lindl. v. roseum hort ex Delch.

C. caudatum Lindl. v. roseum hort ex Delch.
Phragmipedium longifolium (Warscz. \& Rchb.f) Rolfe

C. irapeanum LLlav. \& Lex.

Encyclia aromatica (Batem.) Schlechter 
Cuadro 2. Continúa.

\section{Nombre en Grower's Manual}

E. atropurpureum Willd.

E. atropurpureum Willd. v. roseum Rchb.f.

E. aurantiacum Batem.

E. brassavolae Rchb.f.

E. ciliare L.

E. cnemidophorum Lindl.

E. diotum Lindl.

E. eburneum Rchb.f.

E. endresii Rchb.f. *

E. fragrans $\mathrm{Sw}$.

E. fragrans Sw. v. megalanthum Lindl.

E. mooreanum Rolfe *

E. myrianthum Lindl.

E. myrianthum Lindl. v. album Rchb.f.

E. prismatocarpum Rchb.f.

E. pseudepidendrum Rchb.f.

E. radicans Pav. ex Lindl.

E. stamfordianum Bat.

E. verrucosum $\mathrm{Sw}$.

E. vitellinum Lindl.

Galeandra baueri Lindl.

$G$. dives Rchb.f.

G. harveyana Rchb.f.

G. nivalis hort.

Gongora maculata Lindl.

Ionopsis utricularioides Lindl.

Laelia acuminata Lindl.

Laelia acuminata Lindl. v. rosea hort.

Lycaste cruenta Lindl.

L. deppei (Lodd.). Lindl. v. punctatissima Rchb.f.

L. gigantea Lindl.

L. lasioglossa Rchb.f.

L. schilleriana Rchb.f.

L. skinneri (Batem. ex Lindl) Lindl.

L. skinneri (Batem. ex Lindl) Lindl. v. alba hort (sic)

L. skinneri (Batem. ex Lindl) Lindl. f. amabilis Wms.

L. skinneri (Batem. ex Lindl) Lindl. f. armeniaca Sander

L. skinneri (Batem. ex Lindl) Lindl.

L. skinneri (Batem. ex Lindl) Lindl. f. delicatissima Warner

L. skinneri (Batem. ex Lindl) Lindl.

L. skinneri (Batem. ex Lindl) Lindl. f. gloriosa Wms.

L. skinneri (Batem. ex Lindl) Lindl. f. nigro-rubra hort.

L. skinneri (Batem. ex Lindl) Lindl. f. picturata Warner

L. skinneri (Batem. ex Lindl) Lindl. f. picturata Warner

\section{Pág. Nombre actual}

378 E. cordigera (Kunth in H.B.K) Dressler

378 E. cordigera (Kunth in H.B.K) Dressler

378 Guarianthe aurantiaca (Bat.) Dressler \& Higgins

379 Prosthechea brassavolae (Rchb.f.) Higgins

380 E. ciliare L.

381 E. cnemidophorum Lindl.

382 Encyclia diota (Lindl.) Schlechter

382 E. eburneum Rchb.f.

383 E. endresii Rchb.f.

385 Prosthechea fragrans (Sw.)Higgins

$385 \quad$ P. baculus (Rchb.f.)Higgins

387 Encyclia mooreana (Rolfe) Schlechter

387 E. myrianthum Lindl.

387 E. myrianthum Lindl.

389 Prosthechea prismatocarpa (Rchb.f.) Higgins

389 E. pseudepidendrum Rchb.f.

390 E. radicans Pav. ex Lindl.

392 E. stamfordianum Bat. E. verrucosum $\mathrm{Sw}$.

394 Prosthechea vitellina (Lindl.) Higgins

403 Galeandra batemanii Rolfe

404 G. batemanii Rolfe

$405 \quad$ G. harveyana Rchb.f.

$405 \quad$ G. nivalis Mast.

407 G. leucochila Lem.?

424 Ionopsis utricularioides Lindl.

426 Laelia rubescens Lindl.

426 Laelia rubescens Lindl.

473 Maxillaria cruenta (Lindl.) Lindl.

474 L. deppei (Lodd.). Lindl.

474 Ida gigantea (Lindl.) Ryan \& Oakely

475 L. lasioglossa Rchb.f.

$476 \quad$ Ida gigantea (Lindl.) Ryan \& Oakely?

477 L. skinneri (Batem. ex Lindl.) Lindl.

477 L. skinneri (Bat. ex Lindl.) Lindl. v. alba Dombrain

477 L. skinneri (Batem. ex Lindl.) Lindl.

478 L. skinneri (Batem. ex Lindl.) Lindl.

478 L. skinneri (Batem. ex Lindl.) Lindl.

478 L. skinneri (Batem. ex Lindl.) Lindl.

478 L. skinneri (Batem. ex Lindl.) Lindl.

478 L. skinneri (Batem. ex Lindl.) Lindl.

478 L. skinneri (Batem. ex Lindl.) Lindl.

478 L. skinneri (Batem. ex Lindl.) Lindl.

478 L. skinneri (Batem. ex Lindl.) Lindl. 
Cuadro 2. Continúa.

Nombre en Grower's Manual

L. skinneri (Batem. ex Lindl) Lindl. f. purpurea hort.

L. skinneri (Batem. ex Lindl) Lindl. f. reginae Wms.

L. skinneri (Batem. ex Lindl) Lindl. f. rosea Wms.

L. skinneri (Batem. ex Lindl) Lindl. f. roseo-purpurea Wms. 479

L. skinneri (Batem. ex Lindl) Lindl. f. superba Moore

L. skinneri (Batem. ex Lindl) Lindl. f. vestalis Wms.

L smeeana Rchb.f.

Masdevallia astuta Rchb.f. *

M. attenuata Rchb.f. *

M. costaricensis Rolfe *

$M$. reichenbachiana Endrés ex Rchb. *

M triaristella Rchb.f. *

Maxillaria endresii Rchb.f. *

Miltonia endresii Nicholson *

Moorea irrorata Rolfe

Mormodes colossus Rchb.f.

M. igneum Lindl. \& Paxt. in Paxt. *

Notylia albida Klotzsch

N. bicolor Lindl.

Odontoglossum bictoniense Lindl.

O. cariniferum Rchb.f.

O. cordatum Lindl.

O. krameri Rchb.f. *

O. maculatum LLlav.

O. oerstedii Rchb.f. *

O. oerstedii Rchb.f. v. majus Wms. *

O. rossii Lindl. v. rubescens Lindl.

O. schlieperianum Rchb.f*

O. uro-skinneri Lindl.

O. williamsianum Rchb.f. *

Oncidium ampliatum Lindl.*

Oncidium ampliatum Lindl v. majus hort. ${ }^{*}$

O. bicallosum Lindl.

O. carthaginense (Jacq.)Sw. (sic)

O. cavendishianum Batem.

O. cheirophorum Rchb.f.

$O$. haematochilon Lindl.
Pág. Nombre actual

479 L. skinneri (Batem. ex Lindl.) Lindl.

479 L. skinneri (Batem. ex Lindl.) Lindl.

479 L. skinneri (Batem. ex Lindl.) Lindl.

479 L. skinneri (Batem. ex Lindl.) Lindl.

479 L. skinneri (Batem. ex Lindl.) Lindl.

479 L. skinneri X L. deppei fide Ames \& Correll

482 Dracula astuta (Rchb.f.) Luer

482 M. attenuata Rchb.f.

486 M. reichenbachiana Endrés ex Rchb. ?

$500 \quad$ M. reichenbachiana Endrés ex Rchb.

505 Trisetella triaristella (Rchb.) Luer

$509 \quad$ Maxillaria endresii Rchb.f.

519 Miltoniopsis warszewicsii (Nichols.) Garay \& Dunst.

$528 \quad$ Neomoorea irrorata Rolfe

531 Mormodes colossus Rchb.f.

531 M. igneum Lindl. \& Paxt. in Paxt.

$536 \quad$ Notylia barkeri Lindl.

536 Macroclinium bicolor (Lindl.) Dodson

541 Rhynchostele bictoniensis (Lindl.) Soto A. \& Salazar

543 Oncidium cariniferum (Rchb.f.) Beer

548 Rhynchostele cordata (Lindl.) Soto Arenas \& Salazar

571 Ticoglossum krameri (Rchb.f.) Halbinger

577 Rhynchostele maculata (Lex.) Soto Arenas \& Salazar

581 Ticoglossum oerstedii (Rchb.f.)Rodríguez ex

Halbinger (Lucas Rodríguez in errore!)

581 Ticoglossum oerstedii (Rchb.f.)Rodríguez ex Halbinger

590 Rhynchostele rossii (Lindl.) Soto Arenas \& Salazar

591 Rossioglossum schlieperianum (Rchb.) Garay \&

Kennedy

593 Rhynchostele uro-skinneri (Lindl.)Lindl.

597 Rossioglossum williamsianum (Rchb.) Garay \&

Kennedy

599 Oncidium ampliatum Lindl.

599 Oncidium ampliatum Lindl.

601 Trichocentrum bicallosum (Lindl.) Chase \&

Williams

603 Trichocentrum carthagenense (Jacq.) Chase \& Williams

602 Trichocentrum cavendishianum (Batem.) Chase \& Williams

603 O. cheirophorum Rchb.f.

612 Trichocentrum sp. aff. carthagenense (Jacq.) Chase \& Williams ? 
Cuadro 2. Continúa.

Nombre en Grower's Manual

O. kramerianum Rchb.f.

O. kramerianum Rchb. v. resplendens Rchb.f.

O. leucochilum Batem.

O. luridum Lindl.

O. ornithorhynchum Kunth

O. ornithorhynchum Kunth v. albiflorum Rchb.f.

O. roseum Loddiges

O. roseum Loddiges $v$. superbum hort.

O. sphacelatum Lindl.

O. splendidum A. Rich. (sic)

O. tigrinum La Llave \& Lex.

$O$. warsczewicsii Rchb.f.*

$O$ wentworthianum Batem.

Palumbina candida Rchb.f

Peristeria cerina Lindl.

$P$. elata Hooker

Pescatorea cerina Rchb.f.

Pleurothallis lamprophyllum Rchb.f sic

$P$. leucopyramis Rchb.f.

Polycycnis gratiosa Endrés \& Rchb.f.*

Schomburgkia tibicinis Batem.

Sobralia leucoxantha Rchb.f. *

S. lucasiana hort.

S. macrantha Lindl.

S. sanderae Rolfe

S. wilsoniana Rolfe

Stanhopea ecornuta Lemaire

$S$. graveolens Lindl.

S. saccata Batem.

S. wardii Loddiges

Trichocentrum pfavii Rchb.f.

Trichopilia crispa Lindl.

Trichopilia crispa Lindl. v. marginata Warner

Trichopilia marginata v. lepida Veitch *

Trichopilia suavis Lindl. *

Trichopilia suavis Lindl. v.alba hort.*

Trichopilia suavis Lindl. v. grandiflora hort.*

Warsczewiczella aromatica Rchb.f*

$W$. wendlandii Rchb.f.*
Pág. Nombre actual

615 Psychopsis krameriana (Rchb.) Jones

615 Psychopsis krameriana (Rchb.) Jones

618 O. leucochilum Bat. ex Lindl.

620 Trichocentrum luridum (Lindl.) Chase \& Williams

625 O. ornithorhynchum Kunth

O. ornithorhynchum Kunth v. albiflorum Rchb.f.

629 Trichocentrum sp. aff. carthagenense (Jacq.) Chase \& Williams

629 Trichocentrum sp. aff. carthagenense (Jacq.) Chase \& Williams.

630 O. sphacelatum Lindl.

631 O. tigrinum LLlav. \& Lex. v. splendidum (Rich. ex Duch.) Hooker f.

633 O. tigrinum La Llave \& Lex.

636 O. warsczewicsii Rchb.f.

636 Owentworthianum Batem. ex Lindl.

$640 \quad$ P. candida (Lindl.) Rchb.f.

643 Peristeria cerina Lindl.

$644 \quad$ P. elata Hooker

646 Pescatorea cerina (Lindl. \& Paxt.) Rchb.f.

687 Pleurothallis dolichopus Schlechter

687 Specklinia leucopyramis (Rchb.f.) Luer

689 Polycycnis gratiosa Endrés \& Rchb.f.

708 Schomburgkia tibicinis (Bat.) Batem.

712 Sobralia leucoxantha Rchb.f.

712 S. xantholeuca hort ex B.S.Wms. ?

713 S. macrantha Lindl.

714 S. wilsoniana Rolfe

714 S. wilsoniana Rolfe

721 Stanhopea ecornuta Lemaire

721 S. graveolens Lindl.

723 S. saccata Batem.

725 S. wardii Lodd. ex Lindl.

731 Trichocentrum pfavii Rchb.f.

733 Trichopilia $\mathrm{x}$ crispa Lindl.

734 Trichopilia marginata Henfr.

736 Trichopilia marginata Henfr.

736 Trichopilia suavis Lindl.

736 Trichopilia suavis Lindl.

736 Trichopilia suavis Lindl.

756 Cochleanthes aromatica (Rchb.f.)Schultes \& Garay

757 Cochleanthes aromatica (Rchb.f.)Schultes \& Garay

* Especies recogidas en Costa Rica 


\section{Literatura CitADA}

Aiton, W. 1789. Hortus Kewensis. George Nicol, Londres, 3 vols. 547 p.

Allen, D.E. 1969. The Victorian Fern Craze. A history of Pteridomania. Hutchinson. 83 p.

Allen, P.H. 1949. Orchidaceae in R. Woodson \& R. Schery (eds) Flora of Panamá, Ann. Missouri Bot. Gard. 36(1): 1-132, 36(2): 133-245.

Ames, O. 1937. Orchidaceae in P. Standley (ed.) Flora of Costa Rica. I. Field Mus. Nat Hist. Bot. Ser. 391, pp. 197-306.

Ames, O. \& D.S. Correll. 1952. Orchids of Guatemala. Fieldiana, Bot. 26(1).

Ames, O. \& D.S. Correll. 1953. Orchids of Guatemala. Fieldiana, Bot. 26(2).

Barber, L. 1980. The Heyday of Natural History. Doubleday \& Co., New York. 320 p.

Bernal, J. D. 1983. Science in History. 2. The Scientific and Industrial Revolutions. M.I.T. Press, ${ }^{\text {a }}$. Ed., pp. 509-679.

Coleman, W. 1977. Biology in the Ninthteenth Century. Cambridge. $187 \mathrm{p}$.

Dressler, R. L. 1980. Checklist of the Orchids of Panamá. Monogr. Syst. Bot. Missouri Bot. Gard. 4: I-XXVI.

Dressler, R. L. 1993. Field Guide to the Orchids of Costa Rica and Panamá. Comstock Publ. Assoc. 374 p.

Dressler, R. L. 2003. Orchidaceae in B. Hammel et al. (eds.) Manual de Plantas de Costa Rica. Missouri Bot. Gard.. 3: 1-595.

Hamer, F. 1974a. Orquídeas de El Salvador. I. Min. Educ. Pública San Salvador. 374 p.

Hamer, F. 1974b. Orquídeas de El Salvador. II. Min. Educ. Pública San Salvador. 426 p.

Hamer, F. 1981. Orquídeas de El Salvador. III. Min. Educ. Pública San Salvador. 320 p.

Hamer, F. 1982. Orchids of Nicaragua. 1-2. Icon. Pl. Trop. 7-8, láms. 601-800.

Hamer, F. 1983. Orchids of Nicaragua. 3. Icon. Pl. Trop. 9, láms. 801-900.
Hamer, F. 1984a. Orchids of Nicaragua. 4. Icon. Pl. Trop. 11, láms. 1001-1100.

Hamer, F. 1984b. Orchids of Nicaragua. 5. Icon. Pl. Trop. 12, láms. 1201-1300.

Hamer, F. 1985. Orchids of Nicaragua. 6. Icon. Pl. Trop. 13, láms. 1301-1400.

Hamer, F. 1988. Orchids of Central America. Selbyana 10 (Supl.). $430 \mathrm{p}$.

Hamer, F. 1990. Orchids of Central America. Selbyana 11 (Supl.) 423-860.

Hamer, F. 2001. Orchidaceae in W.D. Stevens et al. (eds.) Flora de Nicaragua. Mon. Syst. Bot. Missouri Bot. Gard. 85: 1612-1860.

Hawkes, A.D. 1965. Encyclopaedia of Cultivated Orchids. Faber, Londres. 602 p.

Morton, A.G. 1981. History of Botanical Science. Academic Press, Londres. 474 p.

Musgrave, T. \& Musgrave, W. 2000. An Empire of Plants. Cassell \& Co., Londres. $191 \mathrm{p}$.

Nordenskiöld, E. 1928. The History of Biology. Tudor Publ., New York. 629 p.

Ossenbach, C. 2003. Breve historia de la orquideología en Costa Rica. Editorial de la universidad de Costa Rica, San José. 97 p.

Pupulin, F. 2002. Catálogo revisado y anotado de las Orchidaceae de Costa Rica. Lankesteriana 4: 1-88.

Schiebinger, L. 2004. Plants and Empire. Harvard, 306 p.

Reinikka, M.A. 1995. A History of the Orchid. Timber Press, Portland. 324 p.

Singer, Ch. 1950. A History of Biology. Schuman, New York. $579 \mathrm{p}$.

Veitch, J. 1906. Hortus Veitchii. Edición privada. Londres. $542 \mathrm{p}$.

Warner, R. \& Moore, Thomas (eds). 1882-1897. B.S. Williams' Orchid Album. Londres, 11 vols.

Williams, B.S. \& Williams, H. 1894. The Orchid Grower's Manual. 7a edición. Londres. 796 p.

Williams, L.O. 1946. Orchidaceae in R. Woodson \& R. Schery (eds.) Flora of Panamá. Ann. Missouri Bot. Gard. 33(1): 1-140, 33(4): 315- 404. 